\title{
EFFECT OF DIFFERENT TREADMILL WALKING TRAINING ON BALANCE AND GAIT IN STROKE PATIENTS
}

leva Eglè Jamontaite ${ }^{1,2}$, Paulius Laguninas ${ }^{1}$, Kristina Bartkiene ${ }^{2}$, Julius Gudavicius², Tadas Margelis²

1 Vilnius University Faculty of Medicine, the Department of Rehabilitation, Physical and Sports Medicine

2Vilnius University Hospital Santaros klinikos, Rehabilitation, Physical and Sports Medicine Centre

Introduction: Balance and walk disorders for patients after cerebral vascular diseases are the major factors restricting the patient's daily activities. Effectiveness of rehabilitation programs with patient's after stroke has been proved by research, but which methods works most effectively and should be widely applied, are still in discussion.

Purpose: Investigate the effect of different treadmill walking training on gait and balance in stroke patients.

Materials and methods: A total of 36 stroke subjects who underwent rehabilitation at Vilnius University Hospital Santaros klinikos, Rehabilitation, Physical and Sports Medicine Centre were recruited in this study. Subjects were randomly assigned into two groups. All subjects received a routine individual physiotherapy two times per day for 30 - 45 minutes, 5 days per week.

The subjects in the 1st group additional received 10-20 minutes gait training of walking forward on treadmill. The subjects in the 2nd group also received additional 10-20 minutes gait training of walking backward on treadmill.

Balance parameters were assessed using Berg Balance Test, gait parameters were evaluated using 10 Meter Walk Test and measuring step length and width.

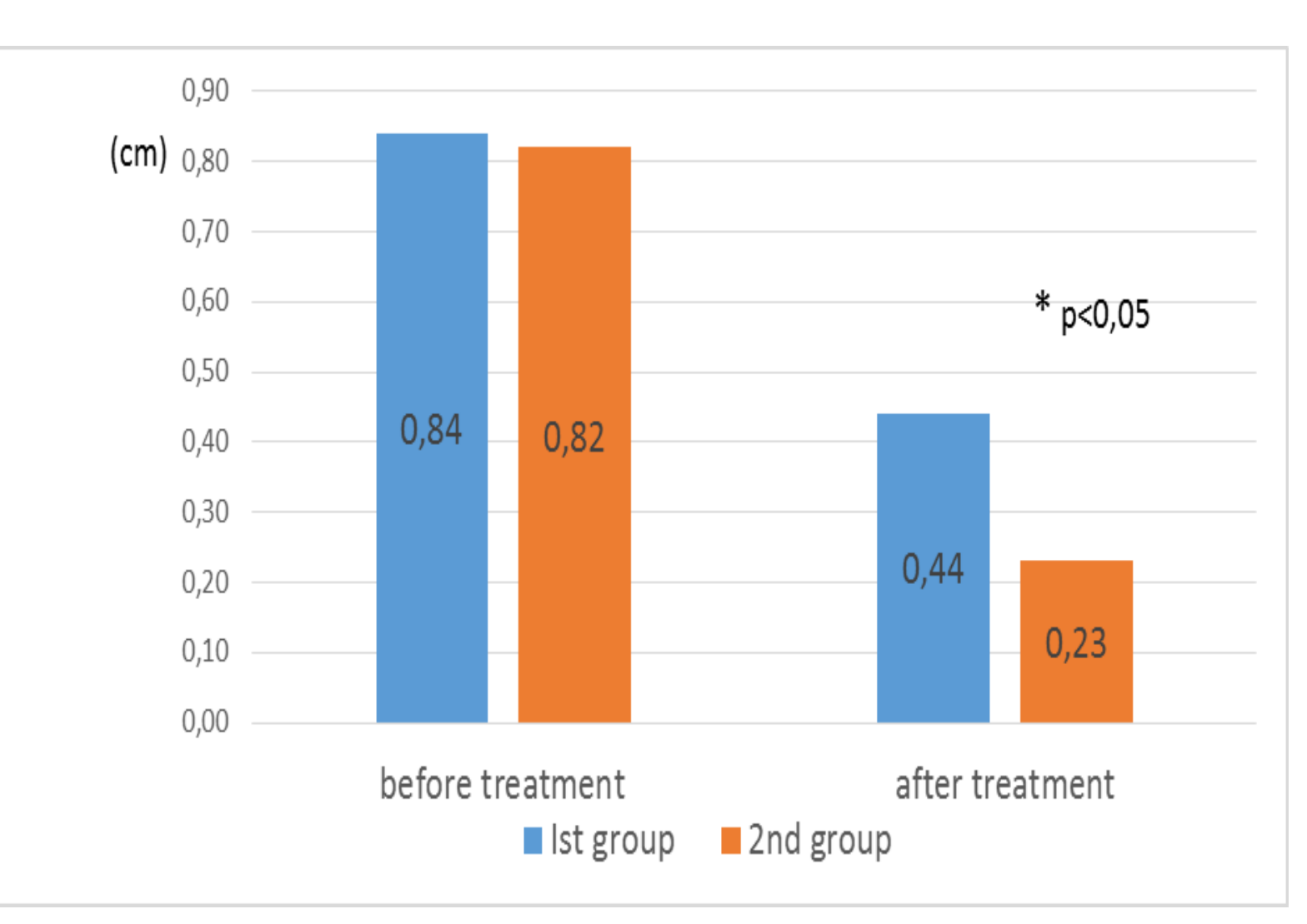

Figure 2. Asymmetry of step width

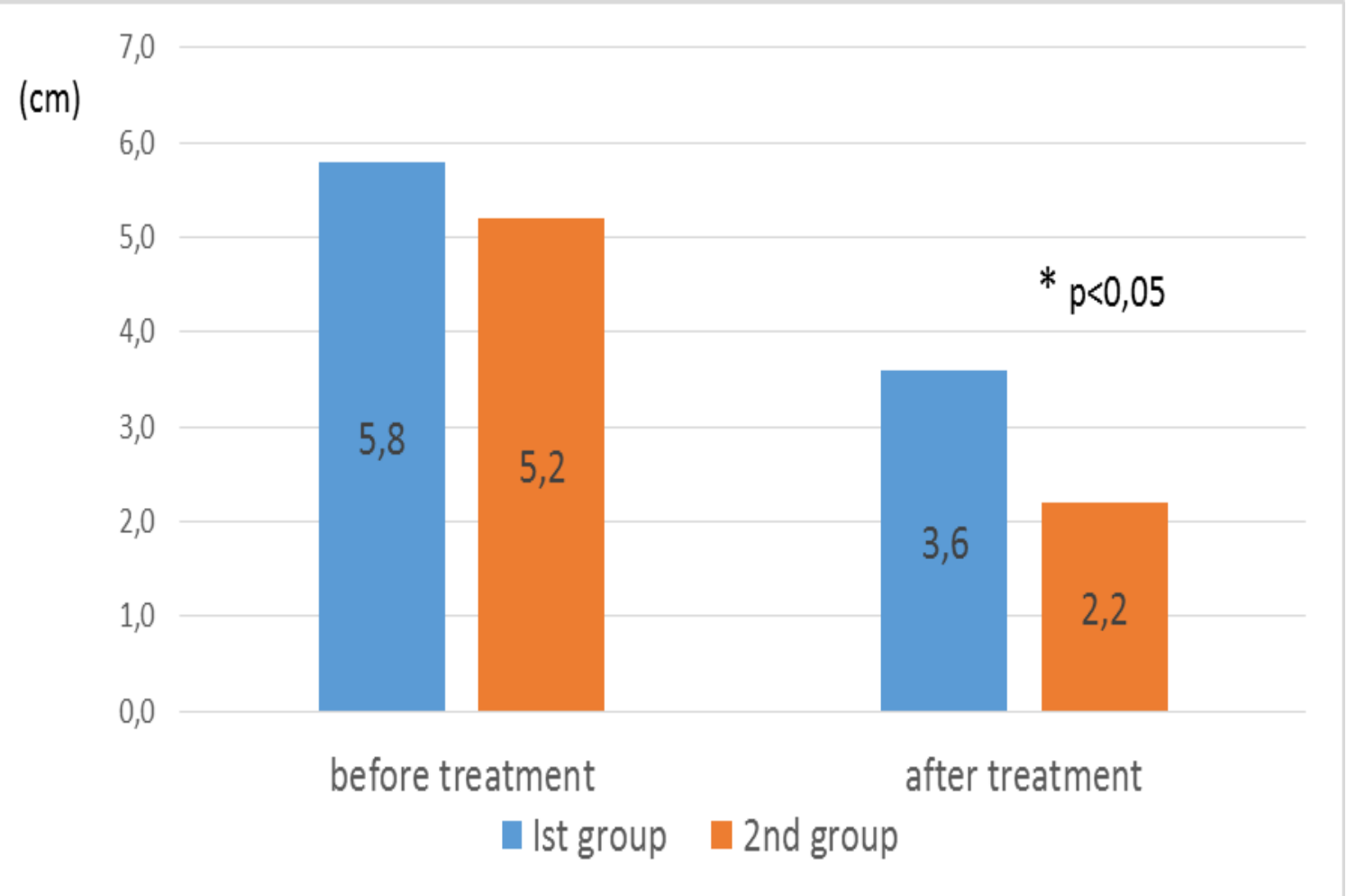

Figure 3. Asymmetry of step length

Results: The results of balance and gait parametres have increased significantly in both groups during the research $(p<0,05)$. However, there was no significantly difference between the groups $(p>0,05)$ comparing the results of balance. Analysis of results of gait parametres tests revealed that in group which received backward training on treadmill significantly more improved gait speed and decreased the asymmetry of step length and width than in the group which received forward walking training on treadmill $(p<0,05)$ (Figures $1-3)$.

Conclusions: The findings demonstrate that backward walking on treadmill was more significantly effective on increasing gait velocity and decreasing asymmetry of step length and width $(p<0,05)$.

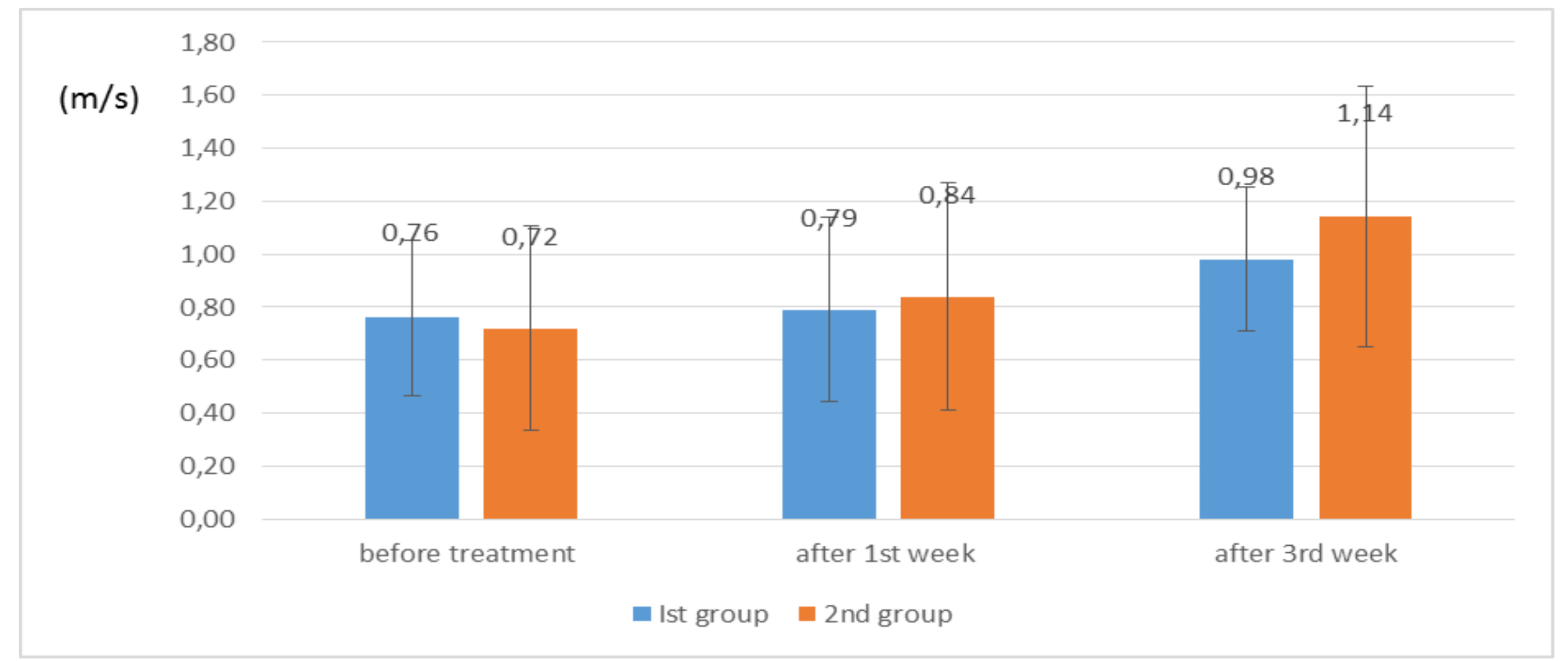

